\title{
PVC Smart Sensing Foil for Advanced Strain Measurements
}

\author{
Alexandre Ferreira da Silva, Anselmo Filipe Gonçalves, Luís Alberto de Almeida Ferreira, \\ Francisco Manuel Moita Araújo, Paulo Mateus Mendes, Member, IEEE, and José Higino Correia, Member, IEEE
}

\begin{abstract}
Monitoring systems can already be found in common applications, from automotive to aeronautics or biomedical. However, the application of these monitoring systems has not yet been a very easy task, especially, at the sensor application step. A smart structure with sensing capabilities would allow to overcome the existing issues in the installation of sensor networks. As the size of this type of systems and the market requirements are significant, an industrial manufacturing process needed to be considered. An integrated solution based in Fiber Bragg Grating sensors embedded in PVC laminates was manufactured by industrial spread-coating process. The resultant structure is a temperature and strain sensitive foil that was characterized in terms of surface structure, optical response and overall performance. The integrated solution behavior had a slope of $0.8 \mathrm{pm}$ by microstrain with a measurement range of $1.2 \mathrm{~mm}$ when the fiber was straight in the foil. Different fiber layouts were also tested and defined the minimum curve radius in $10 \mathrm{~mm}$. The foil also presented a dimensional stability above $\mathbf{9 9 \%}$, ensuring the capability to sustain molding process. A mechanical analysis to evaluate the elongation capabilities and optical response was conducted. Three samples with different sensor positioning were subjected to the test. In the end, a sample, with the sensor in $\mathbf{4 5}^{\circ}$ angle regarding the displacement axis, achieved the best compromise between maximum elongation range $(25 \%)$ and optical response linearity. For the temperature response, a wavelength deviation of $1.7 \mathrm{~nm}$ was obtained for a $100{ }^{\circ} \mathrm{C}$ temperature change.
\end{abstract}

Index Terms-Fiber Bragg gratings, fiber-optic sensor, integrated sensor, sensor for structural monitoring.

\section{INTRODUCTION}

$\mathbf{O}$ PTICAL sensing technologies have associated advantages that make them very attractive in a broad range of applications, from biomedical [1] to civil engineering [2], aeronautics [3], or automotive applications [4]. Optical fiber sensors, in particular, provide low cost solutions, with immu-

Manuscript received September 07, 2009; revised November 11, 2009; accepted November 11, 2009. Current version published April 07, 2010. The work of A. F. da Silva was supported in part by the Portuguese Foundation for Science and Technology under Grant SFRH/BD/39459/2007. This work was supported in part by Project FCT/MIT-Pt/EDAM-SI/0025/2008. The associate editor coordinating the review of this paper and approving it for publication was Prof. Istvan Barsony.

A. F. da Silva, P. M. Mendes, and J. H. Correia are with the Department of Industrial Electronics, University of Minho, 4800-058 Guimaraes, Portugal (e-mail: asilva@dei.uminho.pt; paulo.mendes@dei.uminho.pt; higino. correia@dei.uminho.pt).

A. F. Gonçalves is with TMG Automotive, 4800490 Guimaraes, Portugal (e-mail: filipe.goncalves@tmgautomotive.pt).

L. A. de Almeida Ferreira and F. M. M. Araújo are with FiberSensing Company, INESC Porto, 4470-640 Maia, Portugal (e-mail: luis.ferreira@ fibersensing.com; francisco.araujo@ fibersensing.com).

Color versions of one or more of the figures in this paper are available online at http://ieeexplore.ieee.org.

Digital Object Identifier 10.1109/JSEN.2009.2037014 nity to electromagnetic interference, multiplexing capabilities, and a high degree of miniaturization/integration. Presently, optical fiber sensors offer a high performance alternative, in comparison to standard technologies, either for measuring physical parameters like strain, temperature or pressure, or for performing highly sensitive biochemical analysis [5], [6].

Although the optical fiber sensors present a higher performance when compared to the standard sensing technologies, its application in situ has been one of the major existing issues. In the majority of the cases, the fiber optic sensors are attached to the surface, using epoxy resins [7], woven fabrics [8] or even by welding methods [9]. These approaches present a few concerns due to the fragility of the optical fiber and in optical signal issues as macro- and micro-bendings. These concerns are one of the main barriers for choosing this type of technology. The solution for overcoming these issues have been already proposed in different works by the integration of sensing devices in substrates that are then connected to the host structure [10]-[12]. They were all produced in a laboratory environment, requiring, at this stage, an industrial scale-up for achieving a ready-to-market solution [10]-[12].

The proposed smart structure was designed to be fabricated by already existent industrial processes. Nevertheless, the incorporation of optical sensors creates a few difficulties in eventual sensor maintenance or replacement. Thus, it is proposed the incorporation of optoelectronic instrumentation in standard polymeric foils that can be already found in different products, e.g., automotive and aircraft interior trimmings, wall coverings or in sports suits. The advantages of such smart structure come from the easy applicability of the foils modules in the monitored structure, as well as, easy access for replacement.

Moreover, integrated optical devices are now emerging as the next generation of sensing structures, where virtually any parameter can be determined with high accuracy in a highly miniaturized optoelectronic device [13]. The link of polymer laminates with optical devices and electronics is becoming realistic, enabling its application in any type of surface level.

\section{PVC FOIL WITH FBG SENSOR}

The smart structure based on the integration of strain sensors in polymeric foils is mainly constrained by the fabrication techniques in industrial environment. The majority of the common polymeric foils with customization possibility are based on the spread-coating process.

\section{A. Integration Technology}

Spread-coating technique consists on the deposition of one or more layers of plastisols on a support, such as paper, that is 


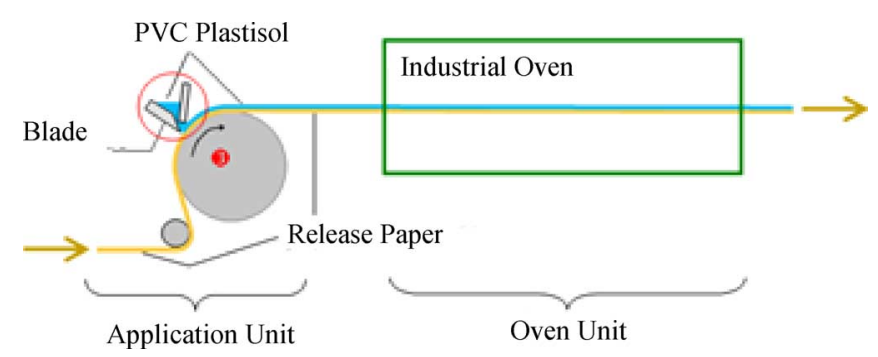

Fig. 1. Layout of industrial spread-coating process.

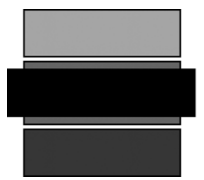

Polymeric layer 3: Final layer (cover)

Polymeric layer 2 with embedded Optical Fiber

Polymeric layer 1: Base layer over Release-paper

Fig. 2. Multilayer structure for embedded optical elements in flexible PVC foil.

cured afterwards in ovens (Fig. 1). Because of its versatility, this technique constitutes an optimal choice for the development of flexible optical sensing foils.

When developing a flexible sensing structure, the need for an easy to apply product becomes evident. In this context, the sensing product should be handled, avoiding damaging the integrated optical sensing elements.

On the other hand, it is important to ensure a good bonding between the sensor and the foil substrate to ensure the minimum sensitivity loss by the polymeric component. Also, the thickness of the whole structure should be the minimum possible, or at least, the distance between the integrated sensor and the host structure should be minimized. This ensures that the existent polymeric layer between the host structure and sensor is reduced, guarantying the transference of the structure behavior stimulus with the minimum interference.

Another requirement for the structure is its ability to be applied in regular and irregular surfaces, enabling a broader application field. This feature requires flexibility and dimensional stability from the sensing structure to sustain some application methods as thermo or vacuum forming.

Consequently, optical fibers and sensors integration should be done by inserting them directly in the carrier matrix and not bonding the optical elements on the carrier surface. With the sensors inside the polymeric matrix is possible to ensure low friction and low damage risk. Furthermore, it guarantees a better bonding of optical fiber with the polymeric matrix, and subsequently a better transfer of stimuli from the host material to the sensor. For this purpose, a multilayer structure approach was chosen (Fig. 2).

Layer \#1 plays the role of a protective skin for the optical fiber. Optical fibers are flexible and can be easily bent but they tend to recover their initial shape. Therefore, it is mandatory to bond the fiber to the substrate over which it is deposited. The use of adhesive polymers is avoided by an intermediate layer (layer \#2). The density and, especially, the whole formulation of this layer are responsible for the fiber adhesion to the carrier and for keeping it steady in its place. Finally a third layer is applied as cover layer.

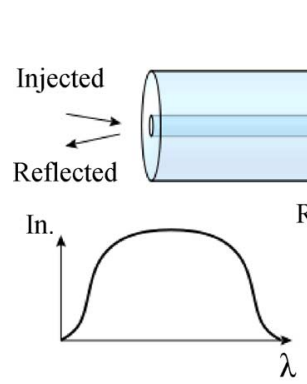

a)

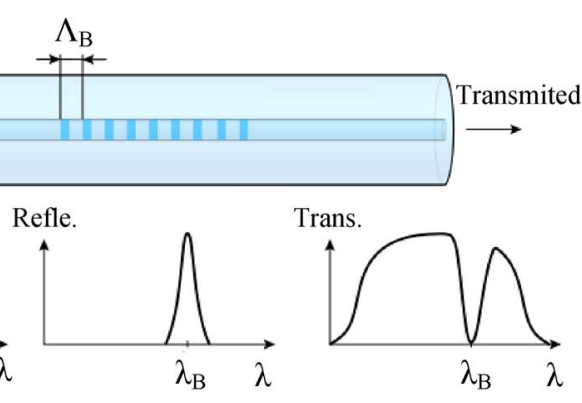

b)

c)
Fig. 3. Illustration of a Bragg sensor working principle: (a) incident spectrum, (b) reflected spectrum, and (c) transmitted spectrum.

\section{B. Optical Fiber Bragg Gratings (FBGs)}

Fiber Bragg Gratings (FBGs) consists in a periodic modulation of the refraction index along the fiber. The produced gratings are typically around $7 \mathrm{~mm}$ long. When an optical beam [Fig. 3(a)] is injected into the fiber with the grating, the wavelength spectrum corresponding to the grating pitch will be reflected [Fig. 3(b)]. The remaining wavelengths will pass through the grating undisturbed, as illustrated in Fig. 3(c) [14].

Since the reflected wavelength depends on the refraction index associated to the fiber and on the diffraction network period, the Bragg wavelength will depend on the physic quantities that may change these parameters, while interacting with the fiber. Consequently, the Bragg sensors are intrinsically sensitive to temperature, axial and transversal deformation, and pressure. Through these sensibilities and especially by the axial deformation, the Bragg sensors can be used for other physical measurements.

Unlike traditional resistance strain gages, FBG sensors are completely passive, offering inherent insensitivity to environmental induced drift and the possibility to obtain, simultaneously, temperature information. The main advantage to use Bragg gratings is its multiplexing potential [14]. Many gratings can be written in the same fiber at different locations and wavelengths tunes. This allows the creation of a strain map along a single fiber. However, since the gratings have to share the spectrum of the light along the fiber, there is a tradeoff between the number of gratings and the dynamic range of the measurements on each FBG.

\section{Polymeric Foil Material}

As the research is focused on the development of a generic manufacturing technology for a flexible optical sensing foil, a polymer matrix with an acceptable average quality and price is desired. Flexible skin-like foils can be made from many different polymers [15].

Polyurethane (PUR) can be one of them with very long durability, high performance in regard to abrasion resistance and flexibility [15]. However, PUR-based foils are one of the most expensive materials in the field of flexible polymeric foils. Polyolefin based artificial skins are a suitable alternative for the required objective, but its softness range and performance, abrasion and flexibility is in general more difficult to adjust [15].

The choice was set on plasticized polyvinyl chloride (PVC), for its general good cost/performance ratio and friendly use during manufacturing processes. The PVC is a highly viscous 
TABLE I

FABRICATION PROCEDURE

\begin{tabular}{|c|l|c|c|c|}
\hline \multirow{2}{*}{ Step } & \multirow{2}{*}{ Operation } & \multicolumn{3}{|c|}{ Condition } \\
\cline { 3 - 5 } & & $\begin{array}{c}\text { Gap } \\
{[\mu \mathrm{m}]}\end{array}$ & $\begin{array}{c}\text { Temp. } \\
{\left[{ }^{\circ} \mathrm{C}\right]}\end{array}$ & $\begin{array}{c}\text { Heating } \\
\text { time [s] }\end{array}$ \\
\hline 1 & Application of PVC-layer 1 & 200 & - & - \\
\hline 2 & Heat-curing of PVC-layer 1 & - & 200 & 60 \\
\hline 3 & Application of PVC-layer 2 & 300 & - & - \\
\hline 4 & Optical fibers insertion & - & - & - \\
\hline 5 & Heat-curing of PVC-layer 2 & - & 200 & 60 \\
\hline 6 & Application of PVC-layer 3 & 400 & - & - \\
\hline 7 & Heat-curing of PVC-layer 3 & - & 200 & 60 \\
\hline 8 & Cooling + manual release & - & - & - \\
\hline
\end{tabular}

mixture that becomes solid on heating. When cooled, the plastisol provides a tough material with good physical characteristics and with a service temperature range of $0^{\circ} \mathrm{C}$ to $125^{\circ} \mathrm{C}$ [16]. Long-term flexibility is the main advantage, which means that the plastisol supports relative motion between host substrates [16]. The major drawback is the cure temperatures that may be too high, limiting their use on specific substrates or applications.

\section{SMART Structure FAbrication}

Werner Mathis ${ }^{\circledR}$ coating equipment was used for the production of laboratory scaled flexible PVC foils with embedded optical fibers. This laboratory technique enables industrial process conditions simulation and is perfectly suitable for industrial scale up.

Since the polymeric layer \#1 is cured, the optical fibers do not penetrate in its core. The layer thickness was approximately $200 \mu \mathrm{m}$. With a thicker skin layer, the whole structure became less flexible, heavy and would contribute for a sensor sensitivity loss. Layer \#2 thickness was defined to $300 \mu \mathrm{m}$, taking into account the $250 \mu \mathrm{m}$ fiber outer diameter. Lower layer thickness would be also feasible from the integration point of view, but the deposited fiber unsteadiness would become more visible. For the final layer, a $400 \mu \mathrm{m}$ thick layer was chosen to ensure enough protection to the optical fiber.

The integration of the optical sensors requires the use of process parameters well-defined during the fabrication. For this specific case, exposing temperature and duration were the main parameters to take into account. The polymer was only able to sustain a maximum of $240^{\circ} \mathrm{C}$ for $150 \mathrm{~s}$, while the fiber could go beyond this value. Thus, the polymer characteristics were the features that constrained the integration process. Most polymeric foils are fabricated in a temperature range of $200^{\circ} \mathrm{C}-220^{\circ} \mathrm{C}$ for $60 \mathrm{~s}$, which is perfectly feasible for this specific case.

The integration procedure that best fit our goal, considering the grounds mentioned above, is presented in Table I.

The fiber Bragg gratings, utilized in these experiments, were produced by FiberSensing. The gratings were written in hydrogen loaded standard telecommunication fiber (Corning SMF $28 \mathrm{e}+$ ) using the phase mask technique and a pulsed Excimer Laser. The length of the gratings is $8 \mathrm{~mm}$ and the resonance wavelength is $1541 \mathrm{~nm}$, corresponding to a refraction index modulation period of the core in the half-micrometer range $(\approx 0.52 \mu \mathrm{m})$, based on the effective refractive index of 1.47.

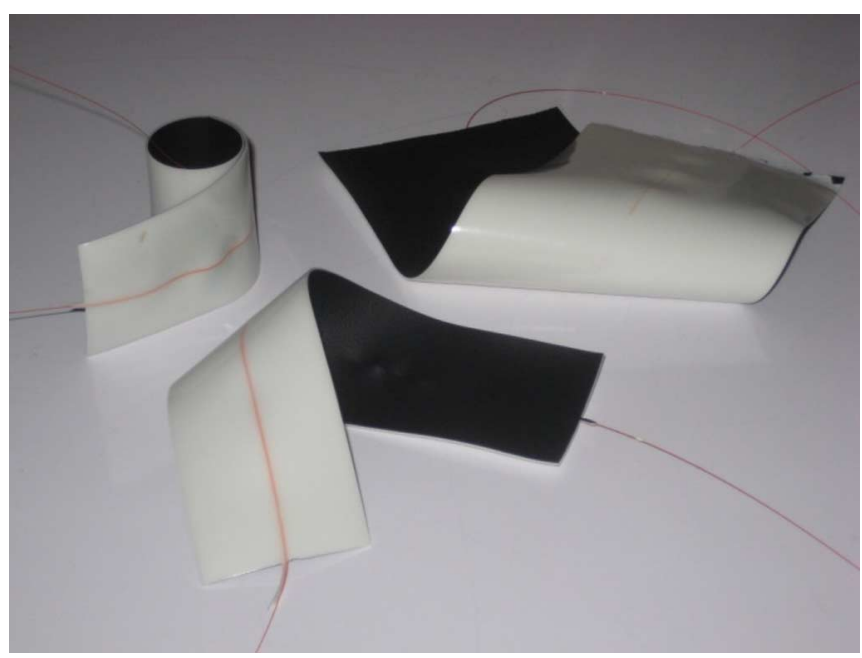

Fig. 4. Fabricated prototypes.
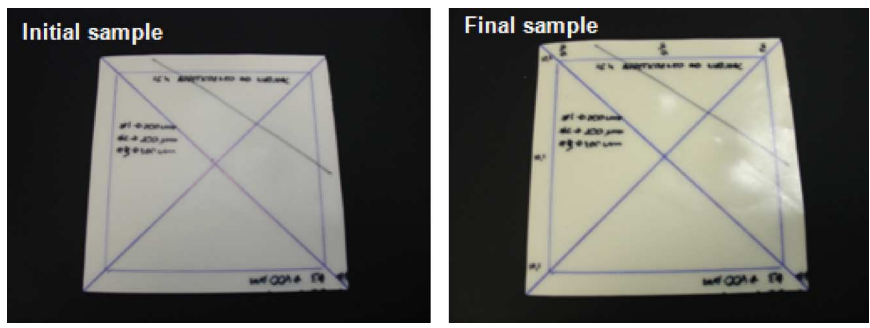

Fig. 5. Dimensional stability test samples, before and after the test.

\section{RESUlts AND STRUCTURE CHARACTERIZATION}

Fig. 4 shows fully functional prototypes produced by spreadcoating process as previously described.

\section{A. Surface Characterization}

By visual inspection, no damage was detected, which is a good indicator of the fabrication processing steps success. By touch, the fiber was not felt, sustaining the idea of a good integration level.

The samples were analyzed at the dimensional stability level. The performed tests evaluated the ability of the structures to retain their shape when submitted to high temperature during a period of time. The initial dimensions were taken at reference marks. Then, the structures were placed in an oven at a specific temperature and exposure time, and, afterwards, reconditioned to room temperature for $1 \mathrm{~h}$. After cooling down, the dimensions were measured again at the same reference marks and compared to the initial values. Two tests conditions were considered, where the samples were first in the oven for $60 \mathrm{~min}$ at $80^{\circ} \mathrm{C}$ and then 1 min to $190^{\circ} \mathrm{C}$.

In both runs, the samples did not present any significant dimension changes. The variations did not reach $1 \%$, which is a very small value and acceptable, in the industry, for PVC foils (Fig. 5).

These results ensured that this PVC matrix provides a secure structure for the molding stage. It was stable enough to be applied in regular and irregular surfaces without presenting major concerning at the formulation level. 


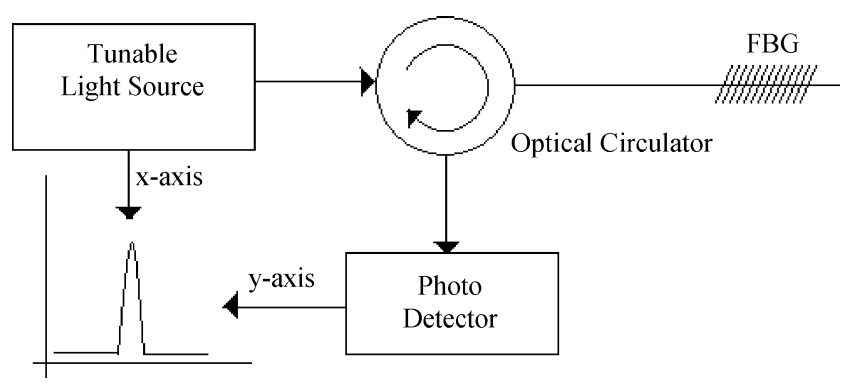

Fig. 6. Interrogation unit setup schematic.

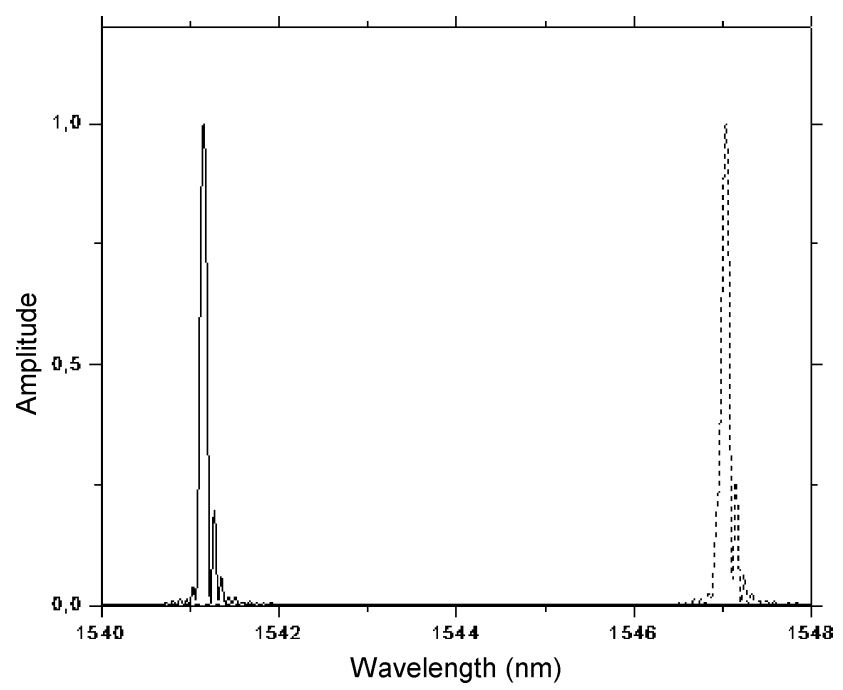

Fig. 7. Reflected spectrum from the FBG sensor for two distinct tensile forces.

Also, the fine-tuning of the PVC matrix properties can be redirected to specific end-uses of the sensing foil. The selected assembly itself can be adapted to special needs. More layers can eventually be added if there is a need for stronger protection of the fibers or a damping effect for the transmitted external stimuli to the optical fibers. Furthermore, one or more layers made of different materials than PVC can be added to the structure. All those changes can be applied to develop a flexible sensing concept according to the needs for the final application.

\section{B. Optical Sensor Characterization}

The optical signature of the Bragg sensor was first acquired by an interrogation unit BraggMETER ${ }^{\text {TM }} 4200$ unit from FiberSensing. This interrogation unit sweeps the $1520-1570 \mathrm{~nm}$ wavelength range and retrieves information about the optical signal amplitude (Fig. 6).

Fig. 7 presents the reflected spectrum of the FBG sensor. The side lobes came from the grating fabrication process, resulting from the radiation transmission function, and not from the integration process. The lobes can be later smoothed by apodization. When stretching the polymeric foil, the embedded FBG sensor followed the deformation and the reflected spectrum suffers a wavelength deviation. When the sample is released, the spectrum returns to its initial position.

\section{Structure Characterization}

Until this stage, data about the individual components of the structure have already been evaluated. In terms of the PVC ma-

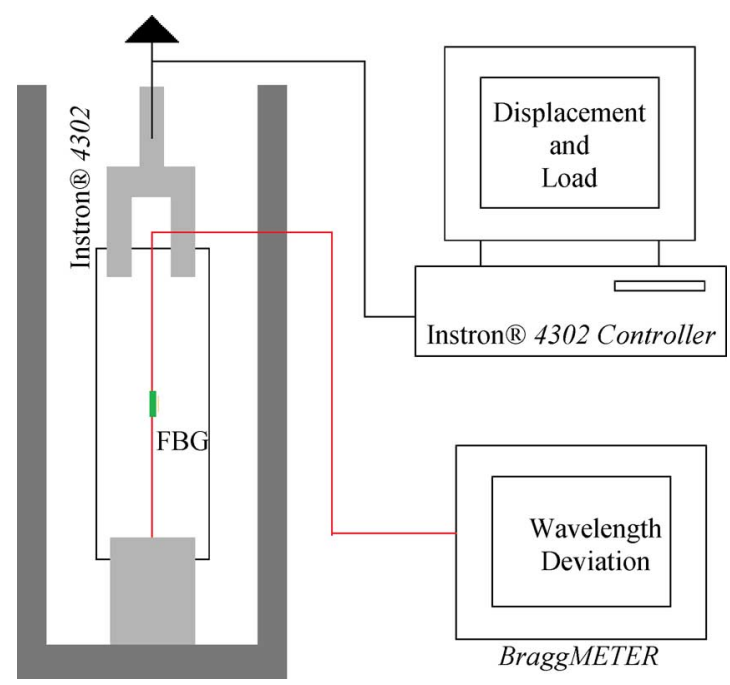

Fig. 8. Testing setup for sensitivity.
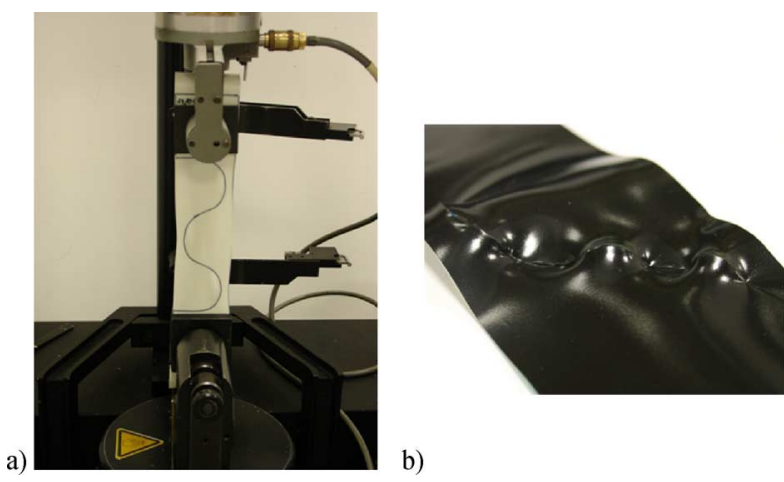

Fig. 9. (a) Sample gripping in elongation-at-break test and (b) detail of the sample surface after the elongation.

trix, the results showed a stable structure, with dimensional stability. The optical sensors were able to sustain the fabrication process without any damage. The optical signal was measured with success.

Also, it is important to evaluate the performance of the overall structure, in order to infer about its sensitivity and integration level. For such goals, the prototype was tested over a setup (Fig. 8) composed by a Instron ${ }^{\circledR} 4302$ testing machine, while the optical signal was being measured by the BraggMETER ${ }^{\mathrm{TM}}$ 4200 unit.

The mechanical study of deformable PVC sensing foils was based on tensile tests [Fig. 9(a)]. The resistive behavior to mechanical stretching of the flexible sensing foils is altered due to the presence of optical fibers. This behavior is dependent of the configuration of the optical fibers or on the optical fiber path in the PVC matrix. These dependencies were better evaluated by the several prototypes that were produced, with different paths of the inserted optical fibers.

When stretching all the considered samples, it was possible to detect a good integration level. In all the samples, the fiber was kept inside the polymeric foil, sustaining the PVC wrinkles due to the stretching in a few specific samples [Fig. 9(b)].

The first set of prototype samples were cut to a $50 \times 100 \mathrm{~mm}$ size strap and two tests were performed. 


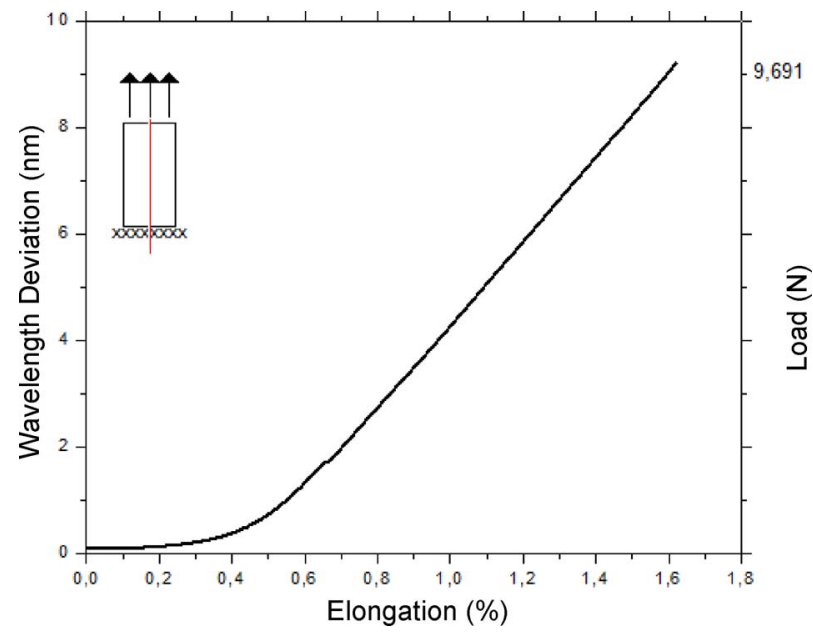

Fig. 10. Wavelength response to applied displacements.

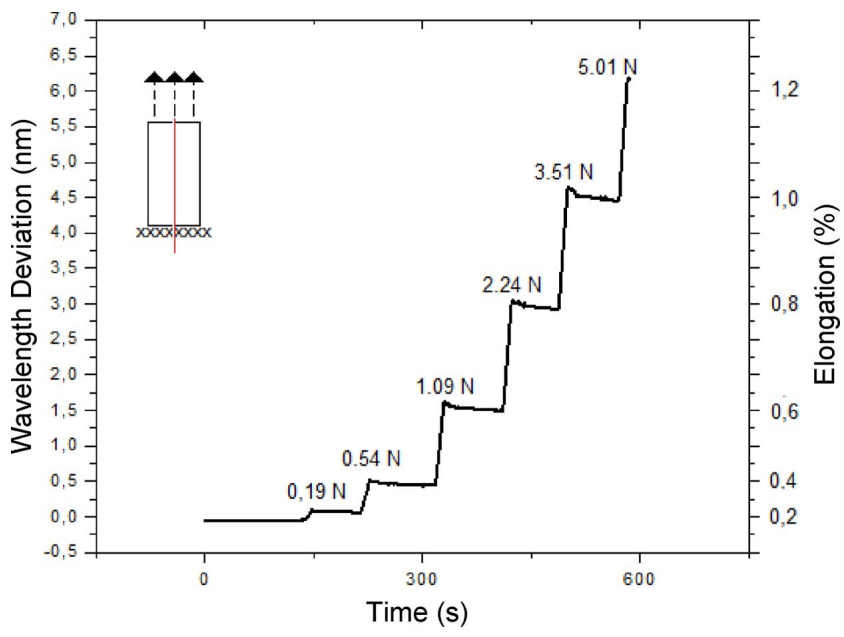

Fig. 11. Wavelength response to applied displacement steps.

In the first test, a displacement was applied at a constant increment rate of $16 \mu \mathrm{m} / \mathrm{s}$. As it is demonstrated over the graph (Fig. 10), the wavelength deviation had a linear behavior above the $0.5 \%$ elongation state. Under $0.5 \%$, the nonlinearity was mainly due to initial stretch state of the sample. At the initial instant, the distance between the grips of the testing machine was lower than the effective length of the foil between the grips due to the foil flexibility.

Besides this fact, the model was able to sustain the $1.62 \%$ stretching, which is $1.62 \mathrm{~mm}$ of displacement in this case. At this time, the fiber was subject to a load of $9.691 \mathrm{~N}$. This displacement of $1.62 \%$ is followed by a wavelength deviation of $9.207 \mathrm{~nm}$, defining the present model response to $0.8 \mathrm{pm} / \mu \varepsilon$ (picometer per microstrain). If this structure was applied to a one meter long steel beam that had been stretched one millimeter, the wavelength deviation that would be measured is $0.8 \mathrm{~nm}$. The determined response value provides a qualitative measurement about the integration quality.

Finally, a displacement was applied in steps of $0.2 \%$ $(200 \mu \mathrm{m})$ and kept at that state during a period of time, in order to evaluate if the fiber slipped over the polymeric foil (Fig. 11). If that happened, the optical signal should decrease while keeping the displacement constant. In Fig. 11, it can be seen
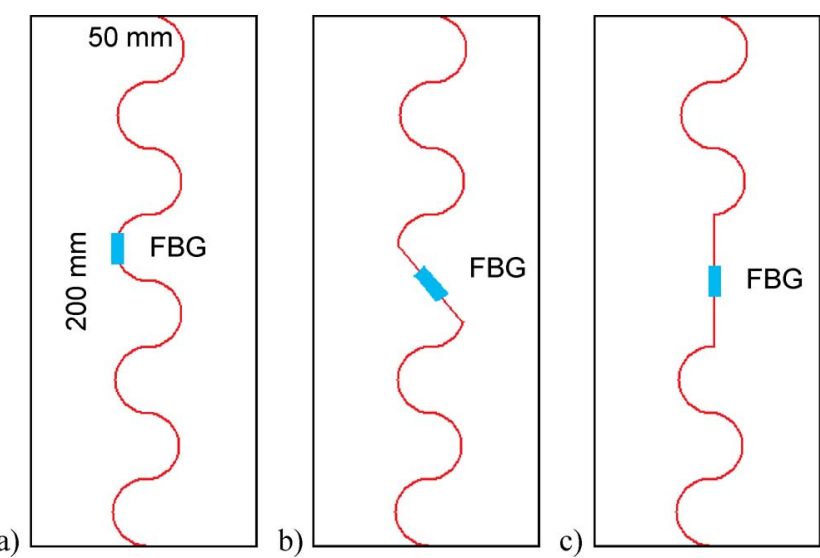

Fig. 12. Illustration of the samples layout considered for the mechanical characterization: (a) Bragg sensor in the curve; (b) Bragg sensor in straight line at $45^{\circ}$ angle; and (c) Bragg sensor in straight line at $90^{\circ}$ angle.

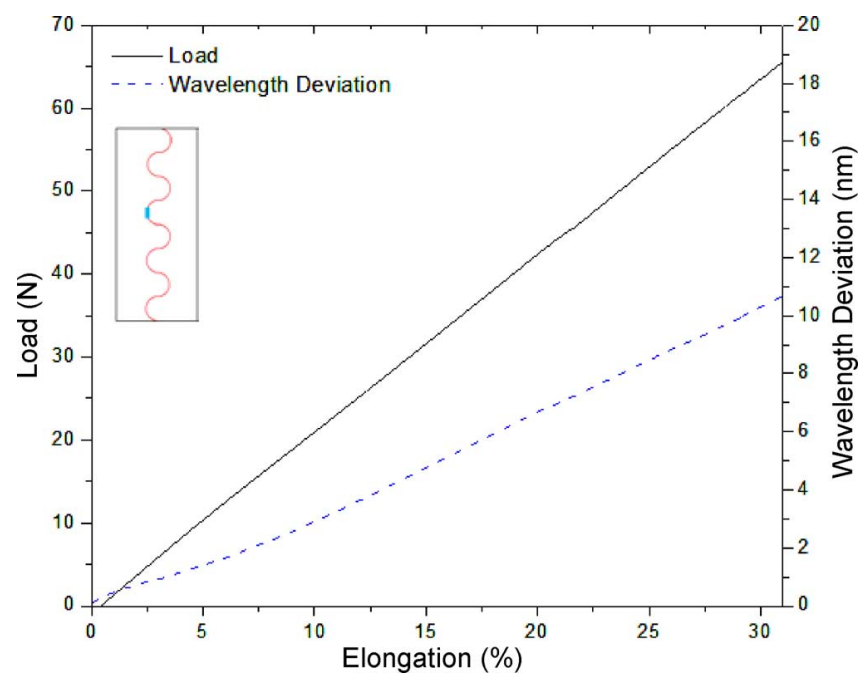

Fig. 13. Sensor response with the Bragg structure in a curve shape.

a little bump after each step, but in seconds the optical signal stayed constant. When stopping the displacement, vibration of the claw was observed and detected by the FBG sensor due to its high sensitivity. The preservation of a constant value ensures that the fiber did not slip and that it was well embedded in the foil.

In the second set of samples, the fiber was mainly set in an "S" pattern with a curve radius of $10 \mathrm{~mm}$. This radius value was considered the minimum that can be successfully done. Lower radiuses $(<10 \mathrm{~mm})$ are not so easy to accomplish during the fabrication process and also introduce losses in the optical signal. Besides the radius value that was kept constant, the variable that was analyzed was the Bragg sensor positioning. Three Bragg layouts were considered relevant (Fig. 12).

All the samples had a similar mechanical behavior. As can be seen from Figs. 13-15, the load lines are very alike, presenting a slight difference in the first values due to the initial stretch state of the structure, which is not the same for all the samples. Also, a quick decrease at the fiber break instant can be seen in Figs. 14 and 15 . The load decreases, momentarily, since there was no more fiber resistance. From this moment on, the resistance was only due to the PVC matrix and the load start to increase again until the collapse of the PVC matrix, which is not represent here 


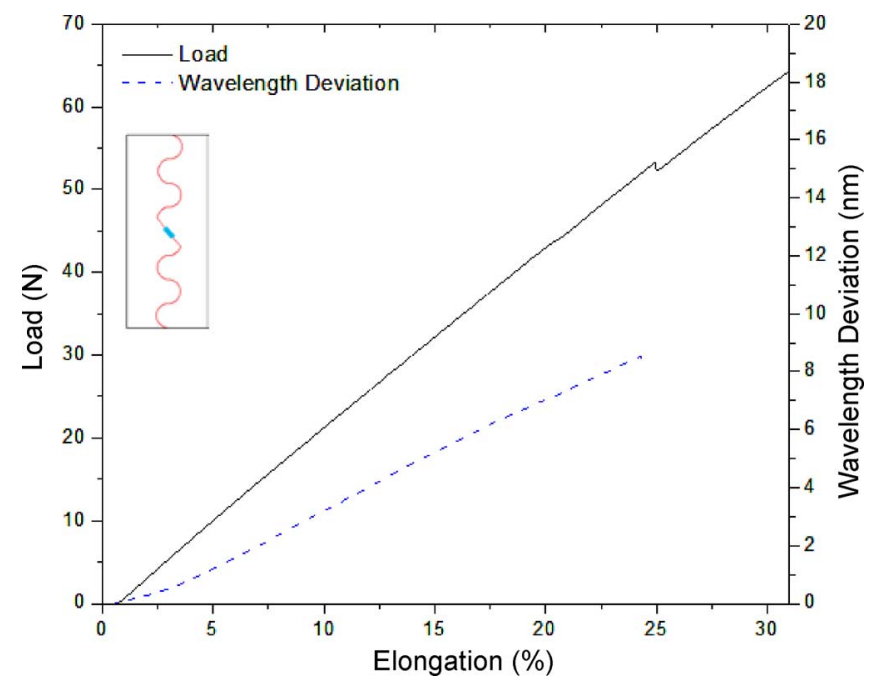

Fig. 14. Sensor response with the Bragg structure in a $45^{\circ}$ angle regarding the elongation axis.

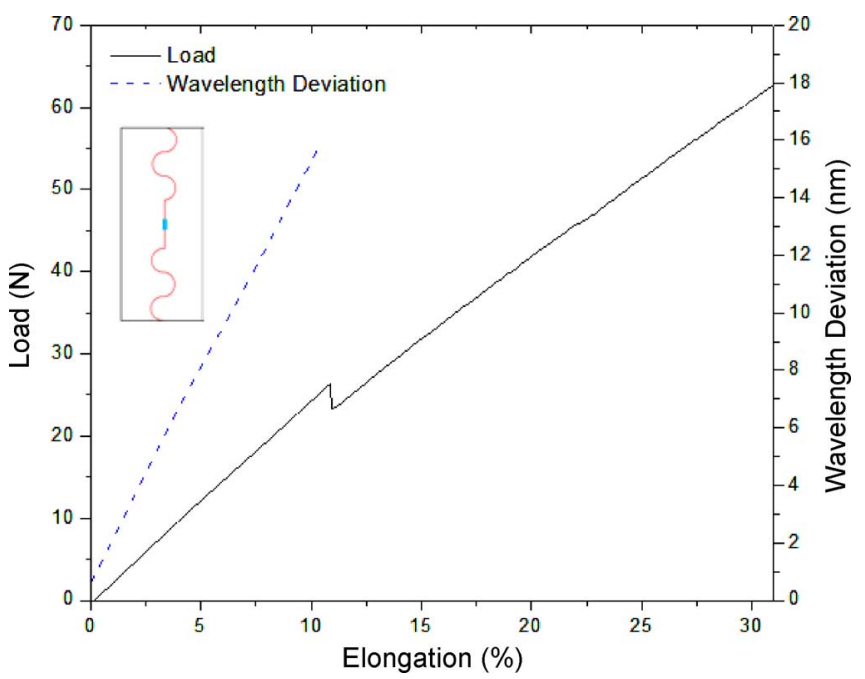

Fig. 15. Sensor response with the Bragg structure aligned with the elongation axis.

since there is no interest in measuring further on, as the optical fiber sensors were not operational anymore.

In terms of optical fiber sensor behavior, there are differences among the three sample types. The maximum elongation is dependent on the Bragg sensor positioning.

As can be seen, the sample with the sensor over the curve (Fig. 13) was the one that was able to reach a higher elongation $(31 \%)$ than the other two. Meanwhile, the samples with the Bragg sensor aligned with the elongation axis (Fig. 15) reached the lowest elongation (10\%). As the middle section is the region where the maximum stress is obtained, the curve sample had the layout that best dealt with that strain concentration.

The sample with the sensor aligned in the same axis as the applied load, was not able to deal, with such success, the mechanical requests, since its positioning does not allow the sensor a significant range of elongation for that specific area.

Another detail that can be seen is the linearity of the optical response. The straight line sample had a linear response due to Bragg sensor orientation, making it only sensitive to axial strain.
The sample with the sensor in a diagonal position (Fig. 14) also had a linear behavior as the one in straight line. Although, due to its orientation, its slope is lower when compared to perpendicular sample. The $45^{\circ}$ angle made the sensor sensitive to the axial and perpendicular components of the structure behavior during the elongation. Consequently, the slope of Bragg response for this sample is low. Besides, this sample was able to sustain an elongation of $25 \%$, thus presenting the best relation between sensor linearity and maximum elongation.

The curve sample (Fig. 13) was the one that was further from a linear response. This structure, in order to sustain the elongation, opens the curves during the stretching providing an elongation range. Although, its elongation is high, its behavior is more complex and consequently non linear.

With respect to the temperature component, the structure presented a response of $1.7 \mathrm{~nm}$ for a temperature variation of $100^{\circ} \mathrm{C}$. The same wavelength deviation would be obtained with $2.5 \%$ of elongation. For higher strain application proposes, the temperature influence can be neglected. However, for the opposite cases, the temperature influence must be taken into consideration. For these cases, at least two FBG need to be used which, one of them is free of strain, in order to measure only the temperature component and remove its influence from the strain data, or filtering may be used to separate both components.

\section{CONCLUSION}

A sensing smart structure based on the integration of FBG sensors in a polymeric foil, using standard industrial fabrication processes was designed. Fully functional prototypes were also developed and analyzed.

The initial structure requirements were fulfilled, taking into account the spread-coating process constraints and overcoming the restraints of the existing solutions.

No damage was detected at the prototype surface. The PVC matrix resulted in a very stable construction with a dimensional stability above $99 \%$. From the optical response of the embedded sensor, the fabrication process did not present any change due to the integration process. The sensor was kept intact during the whole process. The overall structure showed a performance characterized by $0.8 \mathrm{pm}$ per microstrain slope with a measurement range to $1.2 \mathrm{~mm}$ for a fully straight positioning of the FBG. Also, linear sensor response, spectrum shape maintenance during the load application, and measurements repeatability are features that were characterized.

A mechanical study was also conducted, considering the possibility of the structure application in nonhomogeneous surfaces, requiring an elongation capability. A diagonal positioning of the sensor allowed the sample to reach a maximum elongation of $25 \%$ with linear response, presenting the best committing between maximum elongation and linearity response.

The temperature influence may be a concern or not, depending on the magnitude of the strain and the temperature oscillations existent in the host structure. An elongation of $2.5 \%$ had the same wavelength deviation as a temperature shift of $100^{\circ} \mathrm{C}$.

The presented approach enabled a sensing solution that best fits the host structure and the monitoring systems requirements. 


\section{REFERENCES}

[1] G. Wehrle et al., "A fibre optic Bragg grating strain sensor for monitoring ventilatory movements," Meas. Sci. Technol., vol. 12, pp. 805-809, 2001.

[2] Sensing Issues in Civil Structural Health Monitoring. New York: Springer, 2005.

[3] E. J. Friebele et al., "Optical fiber sensors for spacecraft applications," Smart Mater. Struct., vol. 8, p. 813, 1999.

[4] N. Schiller, "Selecting optical detectors for automotive designs," ECN: Electronic Component News, p. 50, Dec. 2004.

[5] A. Dhawan et al., "Plasmonic structures based on subwavelength apertures for chemical and biological sensing applications," IEEE Sensors J., vol. 8, no. 6, pp. 942-950, Jun. 2008.

[6] R. A. Yotter et al., "Sensor technologies for monitoring metabolic activity in single cells-Part I: Optical methods," IEEE Sensors J., vol. 4, no. 4, pp. 395-411, Apr. 2004.

[7] H. F. Lima et al., "Structural health monitoring of the church of Santa Casa da Misericordia of Aveiro using FBG sensors," IEEE Sensors J., vol. 8, no. 7, pp. 1236-1242, Jul. 2008.

[8] A. Grillet et al., "Optical fiber sensors embedded into medical textiles for healthcare monitoring," IEEE Sensors J., vol. 8, pp. 1215-1222, 2008.

[9] R. M. da Costa Marques Pimentel et al., "Hybrid fiber-optic/electrical measurement system for characterization of railway traffic and its effects on a short span bridge," IEEE Sensors J., vol. 8, no. 7, pp. 1243-1249, Jul. 2008

[10] A. K. T. S. Hiroaki Tsutsui and T. Nobuo, "Detection of impact damage of stiffened composite panels using embedded small-diameter optical fibers," Smart Mater. Struct., vol. 13, p. 1284, 2004.

[11] M. Lin and F.-K. Chang, "The manufacture of composite structures with a built-in network of piezoceramics," Comp. Sci. Technol., vol. 62, pp. 919-939, 2002.

[12] D. L. XiaoWen Pan and L. Dongsheng, "Optical fiber sensor layer embedded in smart composite material and structure," Smart Mater. Struct., vol. 15, p. 1231, 2006.

[13] C. Kocabas and A. Aydinli, "Design and analysis of an integrated optical sensor for scanning force microscopies," IEEE Sensors J., vol. 5, pp. 411-418, 2005.

[14] A. D. Kersey et al., "Fiber grating sensors," IEEE J. Lightw. Technol., vol. 15, pp. 1442-1463, 1997.

[15] CES EduPack, 2008. [Online]. Available: http://www.grantadesign. com/education/

[16] E. M. Petrie, PVC Plastisol Adhesives. New York: McGraw-Hill, 2005.

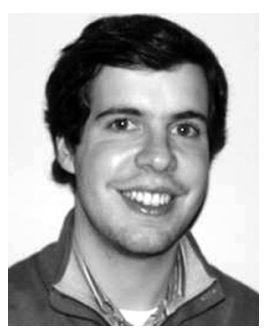

Alexandre Ferreira da Silva received the M.S. degree in biomedical engineering from the University of Minho, Braga, Portugal, in 2007. He is currently working towards the Ph.D. degree at the University of Minho. From 2006 to 2007, he spent six months at RWTH Aachen University, Aachen, Germany, studying alternative sputtering processes in order to evaluate their performance and justify their utilization on electrodes production.

$\mathrm{He}$ is author/coauthor of more than ten international communications.

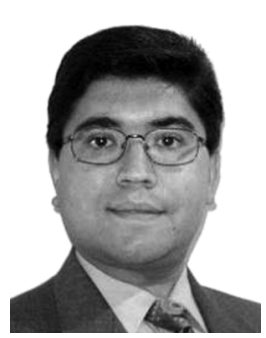

Anselmo Filipe Gonçalves received the Degree in chemistry from the University of Kaiserslautern, Kaiserslautern, Germany, in 1998. In 2007, he attended the Advanced Study Course in Technology Management Enterprise in the MIT-Portugal program.

He is responsible for Innovation and Research with TMG-Automotive, a company of the Textile Group Têxtil Manuel, Gonçalves, Portugal. His professional interests are in flexible polymer foils technology.

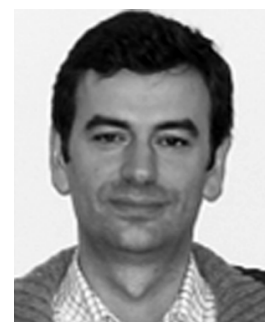

Luís Alberto de Almeida Ferreira graduated in 1991 in applied physics (optics and electronics) and in 1995 received the M.Sc. degrees in optoelectronics and lasers (white-light interferometry and signal processing in optical fiber sensors), both from the University of Porto, Porto, Portugal, and the Ph.D. degree in physics from the University of Porto in 2000 in interrogation of fiber-optic Bragg grating sensors, after developing part of his research work in fiber-optic sensing in the Physics Department, University of North Carolina, Charlotte.

He is the currently an Engineering Manager at FiberSensing, an INESC Porto spin-off company that he co-founded, and that develops, manufactures, and installs advanced monitoring systems based on fiber-optic sensing technology, and that addresses markets such as structural health monitoring in civil and geotechnical engineering, aerospace, and energy production and distribution. $\mathrm{He}$ is also a Senior Researcher at the Optoelectronics and Electronic Systems Unit of INESC Porto, where he develops his main R\&D activity in the areas of fiber-optic sensing and optical communications. He is author/coauthor of more than 100 international communications, papers, and patents in the fields of fiber-optic sensing and fiber-optic communications.

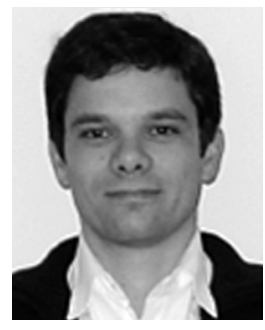

Francisco Manuel Moita Araújo graduated in 1993 in applied physics (optics and electronics) from the University of Porto, Portugal. He received the Ph.D. degree in physics (fiber Bragg gratings) from the University of Porto in 2000.

$\mathrm{He}$ is Product Development Director with FiberSensing, an INESC Porto spin-off company, developing fiber-optic sensors and systems for different markets, such as structural health monitoring. $\mathrm{He}$ is a co-founder of FiberSensing. He is also a Senior Researcher with the Optoelectronics and Electronic Systems Unit of INESC Porto. His main activity research is related with optical communications and fiber-optic sensing. Previous positions included leadership of the Fiber Optic Technologies Unit at MultiWave Networks Portugal, a company developing subsystems for fiber-optic communications, Assistant Professor at the Physics Department of the University of Porto (Faculty of Sciences), and Senior Researcher at the Optoelectronics and Electronic Systems Unit of INESC Porto, were he developed research in the area of fiber-optic technologies from 1993 to 2001. He is author/coauthor of more than 100 international communications, papers, and patents in the fields of fiber-optic sensing and fiber-optic communications.

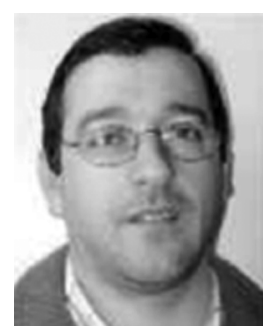

Paulo Mateus Mendes (M'05) graduated in 1995 and received the M.Sc. degree in 1999, both in electrical engineering from the University of Coimbra, Coimbra, Portugal.

From 1999 to 2005, he was a Lecturer with the Department of Industrial Electronics, University of Minho, Minho, Portugal, and since 2005, he has been an Assistant Professor with the Department of Industrial Electronics, University of Minho. He is now involved in research at the Algoritmi Center on waferlevel chip-scale packaging for RF applications, wireless microsystems, antenna miniaturization, biomedical devices, and ambient assisted living technologies.

Mr. Mendes is member of the IEEE Antennas and Propagation Society, IEEE Engineering in Medicine and Biology Society, and the European Microwave Association.

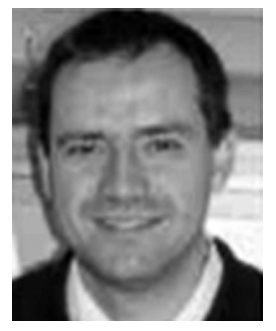

José Higino Correia (S'96-M'00) graduated in physical engineering from the University of Coimbra, Coimbra, Portugal, in 1990. He received the Ph.D. degree in 1999 from the Laboratory for Electronic Instrumentation, Delft University of Technology, Delft, The Netherlands, working in the field of microsystems for optical spectral analysis.

$\mathrm{He}$ is a Full Professor with the Department of Industrial Electronics, University of Minho, Portugal. His professional interests are in micromachining and microfabrication technology for mixed-mode systems, solid-state integrated sensors, microactuators, and microsystems.

Dr. Correia was the General-Chairman of Eurosensors 2003 and MME 2007, Guimarães, Portugal. He is a Member of the IEEE Industrial Electronics Society. 